Canadian

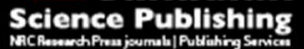

Canadian Journal of Civil Engineering Revue canadienne de génie civil

\title{
Psycho-physiological responses of drivers to road section types and elapsed driving time on a freeway
}

\begin{tabular}{|r|l|}
\hline Journal: & Canadian Journal of Civil Engineering \\
\hline Manuscript ID & cjce-2014-0392.R2 \\
\hline Manuscript Type: & Article \\
\hline Date Submitted by the Author: & 15 -May-2015 \\
\hline Complete List of Authors: & $\begin{array}{l}\text { Kim, Ju-Young; Korea Transportation Safety Authority, } \\
\text { Kim, Jin-Tae; Korea National University of Transportation, Department of } \\
\text { Transportation System Engineering } \\
\text { Kim, Wonchul; Chungnam Development Institute, }\end{array}$ \\
\hline Keyword: & $\begin{array}{l}\text { highways < Transportation, planning < Transportation, surveys < } \\
\text { Transportation }\end{array}$ \\
\hline
\end{tabular}


Psycho-physiological responses of drivers to road section types and elapsed driving time on a freeway

\author{
Ju-Young Kim \\ Researcher, Ph.D. \\ Korea Transportation Safety Authority \\ 5-5 Block Hyuksin-dosi, Yulgok-dong \\ Gimcheon, Gyeongsangbuk-do, Korea \\ TEL:+82-54-459-7242 \\ FAX:+82-54-459-7243 \\ E-mail: micro2@kg21.net \\ Jin-Tae Kim (corresponding author) \\ Assistant Professor, Ph.D. \\ Department of Transportation Engineering \\ Graduate school of Transportation \\ Korea National University of Transportation \\ 157 Cheoldobangmulgkwan-ro \\ Uiwang, Gyeonggi, 437-763, Korea \\ Tel: $+82-31-462-8736$ \\ Fax: +82-31-462-8739 \\ E-mail: jtkim@ut.ac.kr \\ Wonchul Kim \\ Senior Researcher, Ph.D. \\ Dept. of Regional \& Urban Research \\ Chungnam Development Institute \\ 101 Geumheung-dong, Gongju-si \\ Chungcheongnam-do, 314-140, Korea \\ Tel: $+82-41-840-1153$ \\ Fax: +82-41-840-1159 \\ E-mail: iwonchul@cdi.re.kr
}

Words count: $3,958+250 *(7$ figures +9 tables $)=7,958$ 


\begin{abstract}
This paper addresses drivers' psycho-physiological condition under the influence of various freeway section types and elapsed driving times. The authors analyzed the electroencephalogram (EEG) signals $(\alpha, \beta$, and $\theta)$ of 51 drivers on a freeway in Korea. The findings show that the driver's workload increases in tunnels and on left-curved sections, and that his or her concentration and response ability decrease after 60 minutes of elapsed driving time. The $\beta / \alpha$ ratios of EEG signals were found to be most effective in detecting differences in psycho-physiological responses. The results can help to promote safety on freeways by encouraging drivers to take rests every hour.

Key words: electroencephalogram, psychology, workload, freeway, safety, elapsed driving time
\end{abstract}

\title{
1. Introduction
}

The causes of traffic accidents on freeways include faulty conditions of vehicles, drivers, and highways, and their combinations (Garber and Hoel 2009; Evans 1991; Forbes; 1975). Recent statistics show that the number of traffic accidents on freeways in Korea reached 3,550 in 2013 (Korean National Police Agency 2013). Over 70\% (2,609) of these were caused by drivers' mistakes that primarily relate to the increase of distraction and the reduction in concentration due to aspects such as aggressive driving, cell phone use, driving under the influence, and driving while drowsy. One of the causes involved is likely to be driving for a long period of time without a rest. This has motivated campaigns to suggest drivers take regular breaks when driving longer distances in order to reduce fatigue and maintain their level of concentration.

The number of studies that measure human psycho-physiological conditions such as comfort, workload, and concentration has increased in various fields of engineering (Campbell 2008; Scheuchenpflug 2008). Many studies have shown that signals perceived through a person's visual and auditory sensory nerves are useful measures to quantify human psycho-physiological responses to environmental conditions (Bridger 1995; Campbell 2008; Scheuchenpflug 2008). In the field of transportation engineering, topics related to drivers' psycho-physiological responses within the context of freeway safety have been studied. This paper presents a study conducted to quantify the psychophysiological responses of drivers to varying conditions of freeway sections and elapsed driving time. 
The objective of this study is to encourage discussion on effective safety measures and safety campaign programs by providing scientific findings on how human factors are affected by freeway section types and elapsed driving time. This includes findings on (1) the workload that drivers experience with different types of freeway sections, (2) the reduction in drivers' concentration with increasing driving duration, and (3) a recommended maximum length of driving episodes.

\section{Background}

Humans' psycho-physiological responses relate to the central nervous system (CNS), which transfers signals between the brain and the muscles. CNS measures have been used in various studies, most often by applying electroencephalograms (EEG) that capture bio signals from the brain. EEGs have been widely used in medical science and engineering due to their sensitivity to small changes in a person's psychological condition (Niedermeyer and Silva 2005; Wierwille and Eggemeier 1993; Helander 1975; Katsuki 2003). It is possible to quantify a person's level of comfort and distraction by referring to the different sensory inputs captured in an EEG, since specific parts of the human brain control specific functions. For example, the rear part of the brain deals with visual information, whereas the left and right sides of the brain deal with auditory information. Changes in a person's psycho-physiological responses can also be obtained from EEGs.

An EEG depicts measurement in several waves, termed $\alpha, \beta, \theta$, and $\delta$. Fig. 1 depicts the shapes of the signals in excited, relaxed, drowsy, and sleeping states. The $\alpha$ wave is a rhythmic oscillation that occurs at a rate of 8 to $13 \mathrm{~Hz}$, and at a magnitude of about 20 to 60 millionths of a volt. The $\alpha$ wave is dominantly produced when a person is in a state of relaxation, calmness, and stability. As soon as the individual becomes either mentally or physically active, the spectrum (the difference between the highest and the lowest readings) of the $\alpha$ wave decreases and disappears. The $\beta$ wave is an irregular oscillation occurring at a frequency of 14 to $30 \mathrm{~Hz}$, and with an amplitude of approximately 2 to 20 millionths of a volt. The $\beta$ wave is dominantly produced when a person is involved in mental or physical activities. As soon as the individual becomes relaxed (calm and stable), the spectrum of the $\beta$ wave decreases and disappears. The $\theta$ wave is a relatively less common type of brain rhythm that occurs at about 4 to $7 \mathrm{~Hz}$, with amplitude ranging from 20 to 100 millionths of a volt. It has been reported that the 
$\theta$ wave can be found more frequently in the spontaneous EEG recordings of children than those of adults (Walter 1953).

Brookhuis et al. (1994) suggested using the "relative energy parameter $\left(P_{r e}\right)$ " as a measure of human psycho-physiological conditions and defined it as a function of the spectra $\alpha, \beta$, and $\theta$. The $P_{r e}$ function is as shown in Equation 1.

$$
P_{r e}=\frac{\theta_{\text {spectrum }}+\alpha_{\text {spectrum }}}{\beta_{\text {spectrum }}}
$$

The spectra of $\alpha$ and $\beta$ represent a person's level of relaxation and activity, respectively. When a person is under pressure by a heavy workload, the spectra of the $\alpha$ and $\theta$ waves decrease, while the spectrum of the $\beta$ wave increases, causing the $P_{r e}$ parameter to decrease. Conversely, when a person relaxes, the $P_{r e}$ value increases.

Kim (1998) and Park et al. (1999) indicated that $\alpha$ and $\beta$ are the major EEG signals directly related to the workload of drivers, while the $\theta$ wave is less important. These $\alpha$ and $\beta$ waves inversely and simultaneously increase and decrease in driving conditions, respectively. They suggested using the $\beta / \alpha$ ratio as the measure of a driver's psychophysiological condition. High values of the ratio indicate a high workload, since a driver is mentally or physically active. The value becomes less significant in drowsy driving, as the activation of the $\theta$ wave significantly affects the $\alpha$ and the $\beta$ waves. The $\beta / \alpha$ function is as shown in Equation 2.

$$
\beta / \alpha=\frac{\left(\beta_{\text {spectrum }}-\alpha_{\text {spectrum }}\right)}{\alpha_{\text {spectrum }}}
$$

Equation 2 fundamentally differs from Equation 1 as it excludes the $\theta$ wave from the equation. However, no studies have yet deterministically compared the two measures in terms of their performance. Both measures were initially employed in this study to identify the better measure and this will be discussed in detail later in this paper.

\section{Methodology}

Drivers' EEG data were collected in a field experiment. The test scenario and the data collection method were designed to investigate drivers' psycho-physiological condition under the influence of different freeway section types and elapsed driving times. A test vehicle was developed to collect the EEG data from drivers in real time. The following subsections describe the data collection method, the equipment, the test drivers, and the test conditions used in the study. 


\subsection{Test equipment}

A driver's behavior and response detecting vehicle (DBDV) was developed to simultaneously collect information on (1) drivers' psycho-physiological condition and (2) freeway traffic and geometric conditions. Fig. 2 depicts the DBDV and its component systems. The DBDV is equipped with video systems utilizing three closed circuit television (CCTV) cameras that record streaming data on freeway and driver conditions. Two of the cameras are placed on top of the vehicle to record the traffic and geometric conditions, while the third is placed on the dashboard to record drivers' facial information. The DBDV directly records speed data from the tachometer and collects drivers' psycho-physiological signals through the EEG data collection system.

\subsection{Data collection}

Bio-signals were collected and recorded from the surface of the driver's head through electrodes connected to an EEG cap (see Fig. 3). Lamm et al. (1999) reported that, although most senses are used in driving, a driver detects as much as $90 \%$ of all information through visual and auditory sensors. Fig. 4 depicts the signal collection points on the EEG cap. Data signals were recorded from two different lobes of the brain: the occipital lobe, consisting of $\mathrm{O} 1$ and $\mathrm{O} 2$ (the points used to collect the effects of visual sensory inputs on a driver's psycho-physiological condition); and the temporal lobe, consisting of T3, T4, T5, and T6 (the points used to collect the effects of auditory sensory inputs). Low-pass and high-pass filters were set as band limits to control the aliasing of signals. This study utilized $256 \mathrm{~Hz}$ as the EEG sampling frequency, and 0.5 $\mathrm{Hz}$ and $300 \mathrm{~Hz}$ for the low-pass and high-pass filter values, respectively, as used in artifact detection and correction.

\subsection{Participants (Test Drivers)}

Fifty-one drivers participated in the field experiment. They consisted of 39 males and 12 females, ranging from 25 to 40 years old. The characteristics of the test drivers are as shown in Table 1. They were unpaid public volunteers selected by interview. All participants had more than 3 years driving experience. Drivers with health problems that might affect the psycho-physiological signals were excluded through the interview. The selected participants were asked to have a normal night of sleep before the test and to avoid smoking and drinking coffee on the test day to eliminate undesirable elements 
that might affect their psycho-physiological signals. People who failed those requirements on the test day were also excluded.

\subsection{Freeway Sections}

The participants were asked to make a round trip $(176 \mathrm{~km})$ on a defined test section of the Youngdong freeway in Korea. This is a six-lane freeway (three lanes in each direction) with a speed limit of $100 \mathrm{~km} / \mathrm{h}$. The test section consisted of several subsections, as presented in Table 2. It was necessary to define the buffer areas between the subsections to avoid confusion between drivers' psycho-physiological signals affected by consecutive freeway section types and to ensure the EEG signal data groups would be mutually independent. Excluding these buffer areas, the length of the test section where the EEG signal data were collected was $88 \mathrm{~km}$.

\subsection{Test Designs}

At the time of the experiment, the participants were informed of the test procedure and possible risks. They were asked to not talk during the test, to not listen to the radio, and to not touch the electrodes attached to their heads (see Fig. 3) to avoid disturbing the recording of the psycho-physiological data. The surrounding air conditioner of the DBDV was switched on during the test to stabilize the indoor temperature and to prevent drivers from possibly becoming drowsy due to increasing temperature. They were asked to relax for five minutes prior to the test to initialize their EEG signals by monitoring their displayed biological signal patterns. The total time period for data collection per participant was limited to two hours because, in outdoor test environments, the EEG gel used to attach the electrodes to participants' heads then starts to lose its function.

All data were collected during a non-peak period, from 2:00 p.m. to 4:00 p.m., to control the traffic conditions such as the level of service (LOS) C. All data were collected on sunny weekdays to ensure maximum consistency of illumination and noise levels during the test. To maintain consistent driving conditions, the test drivers were asked to only use the rightmost lane.

\subsection{Data Analysis}

The spectra of the EEG waves were analyzed based on the relative power value obtained after normalization, as shown in Equations 3 and 4: 


$$
\begin{gathered}
R_{\text {power }}=\frac{E_{\text {power }}}{\sum A_{\text {power }}} \\
N_{p}=\frac{P_{\text {driving }}}{P_{\text {resting }}}
\end{gathered}
$$

where $R_{\text {power }}=$ relative power value, $E_{\text {power }}=$ specific power value, $A_{\text {power }}=$ all power values, $N_{p}=$ Normalized value, $P_{\text {driving }}=$ biological signal value in a driving condition, and $P_{\text {rest }}=$ biological signal value in a resting condition.

\section{Results and Analysis}

The spectra of the EEG waves were calculated based on the Fast Fourier Transform method (James, 2011). Table 3 presents the computed values categorized according to freeway section type, while distinguishing between visual effects (occipital lobe) and auditory effects (temporal lobe). Based on the spectra of the EEG waves, drivers' psycho-physiological conditions were analyzed in relation to freeway facilities types and elapsed driving times. The $P_{r e}$ and the $\beta / \alpha$ measures were separately applied to determine the better measure of the drivers' psycho-physiological condition in response to visual and auditory sensory inputs.

\subsection{Initial Analysis of Driver's Workload Related to Freeway Section Type}

The workloads experienced by drivers due to the visual and the auditory effects of each freeway section type were compared.

\subsubsection{Visual effects}

Fig. 5 presents the drivers' workload based on the visual effects of different freeway section types. Figs. 5(a) and 5(b) show the observed measures of $P_{r e}$ and $\beta / \alpha$, respectively. Fig. 5(a) shows that the $P_{r e}$ value was high when drivers were at rest but reduced by half in driving conditions to a value of around 2.0, regardless of the type of freeway section. This implies that drivers experience a certain amount of workload caused by visual effects while driving. Careful statistical analysis is needed to identify differences in the visual effects of freeway section types.

Fig. 5(b) shows that the $\beta / \alpha$ value was negative in a resting condition but positive during driving. This concurs with the above finding that drivers experience a certain amount of workload due to visual effects, regardless of the section type. In addition, the values found on the right-curved and tunnel sections were higher than those in the other 
sections. This indicates that the workload due to visual effects on the right-curved and tunnel sections is considerably higher than elsewhere. Additional analysis is thus needed.

\subsubsection{Auditory effects}

Fig. 6 presents the drivers' workload based on the auditory effects of each type of freeway section. Fig. 6(a) shows that the $P_{r e}$ value reduced by half in all driving conditions, when compared to the value at rest. This implies that drivers experience a certain amount of workload due to auditory effects, regardless of the type of freeway section. The same is found with the observed $\beta / \alpha$ values (Fig. 6(b)), warranting further statistical analyses. Fig. 6(b) shows that the $\beta / \alpha$ value in tunnel sections was four times higher than that on other sections. This indicates that the workload increased considerably in tunnel sections due to auditory effects. Additional analysis is thus needed.

\subsection{Initial Analysis of Driver's Workload Related to Elapsed Driving Time}

Fig. 7 presents the drivers' workload due to elapsed driving time. Fig. 7(a) shows that the $P_{r e}$ value was over 4.7 when drivers were at rest, while it decreased to between 2.5 and 2.8 when they were driving. The $P_{r e}$ values, however, did not change significantly as driving time increased. Fig. 7(b) shows that at the start of test driving, the $\beta / \alpha$ value was relatively high (0.929). This might reflect the heightened state of alertness that drivers are expected to experience due to their unfamiliarity with the test vehicle and conditions, since the data also show the value reduced to a certain level (0.838) after 30 minutes of driving, which is a period of time at which drivers may reasonably become accustomed to the new situation. After 30 minutes, the $P_{r e}$ value gradually increased to 0.920 as the elapsed travel time increased. This may reflect the gradual effect of the $\theta$ wave and indicate driving in an increasing state of drowsiness. Further statistical analysis is therefore needed.

\subsection{Additional Analysis of Driver's Workload Related to Freeway Section Type}

The effects of different freeway section types on a driver's workload were further tested using Analysis of Variance (ANOVA) to assess the dissimilarity and the Duncan's non-parametric multiple range test to categorize this dissimilarity. The Duncan's test was only applied when the ANOVA test results showed the existence of a dissimilarity. 


\subsubsection{Visual effects}

Table 4 summarizes the results of the ANOVA tests conducted with the $P_{r e}$ and $\beta / \alpha$ measures related to visual effects at the 0.05 significance level. The results indicate that, according to both measures, there should be at least one section type causing different visual effects than others. Therefore, Duncan's tests were performed to ascertain which types of freeway section were dissimilar. Table 5 shows that the $P_{r e}$ value differs between the rest condition and the driving conditions, as it is statistically categorized into a single group. This supports the notion that drivers experience psychophysiological workload through visual effects when they drive, regardless of the freeway section types they encounter.

Table 5 shows that the $\beta / \alpha$ values found on the left-curved and tunnel sections of the freeway statistically differed. This could be due to several reasons: the drivers' sight distance is geometrically limited on left-curved and tunnel sections; drivers cannot predict the alignment of the freeway ahead; and the lighting conditions on tunnel sections is usually gloomy. These visual effects are likely to increase drivers' psychophysiological workload.

\subsubsection{Auditory effects}

Table 6 summarizes the results of the ANOVA tests conducted with the $P_{r e}$ and $\beta / \alpha$ measures related to auditory effects at the 0.05 significance level. The results show that again, according to both measures, at least one section type should cause different auditory effects than the other sections. Hence, Duncan's tests were performed as presented in Table 7.

Table 7 shows that the $P_{r e}$ value differs between the resting condition and the driving conditions, being statistically categorized into a single group. This indicates that drivers experience psycho-physiological workload through auditory effects when they drive, regardless of the freeway section type. It also shows that the $\beta / \alpha$ value reflecting the auditory effect on tunnel sections statistically differs from other section types, implying the high noise drivers experience on tunnel sections increases their psychopsychological workload. 


\subsection{Additional Analysis of Driver's Workload Related to Elapsed Driving Time}

Table 8 presents the results of the ANOVA tests conducted with the $P_{r e}$ and $\beta / \alpha$ measures related to the effects of elapsed driving time at the 0.05 significance level. It was hypothesized that drivers' concentration level should decrease gradually after a certain period of driving, as increasing fatigue is likely to affect their psychophysiological condition and driving workload.

The test results reveal that only the $\beta / \alpha$ measure verifies the effects of elapsed driving time on workload. This concurs with earlier findings indicating that driving time affects drivers' workload (Shin 1995; Tijerina 1996). Duncan's non-parametric test was performed to assess the threshold value of the elapsed driving time. Table 9 shows that, after a driving time of more than sixty minutes, the $\beta / \alpha$ values statistically differed from the other values. This implies that the workload on drivers is likely to increase after 60 minutes of continuous driving. As their concentration and response abilities may imperceptibly decrease, they will become more easily distracted and stolid in response to the demands of driving.

\section{Discussion}

The changes in drivers' psycho-physiological condition were studied based on EEG signals to assess the workload experienced by drivers under the influence of different types of freeway sections and elapsed driving time. The test drivers were asked to drive on a rural freeway in a light traffic condition (LOS C) with low percentages of heavy vehicles. The types of freeway sections considered in this study included tunnel, interchange, on- and off-ramps, straight tangent, and right- and left-curved sections. The findings show that drivers' workload increases in tunnels due to both visual and auditory effects. Moreover, it was found that left-curved sections introduce high levels of workload due to visual effects, which is most likely due to the difficulty drivers experience observing the downstream alignment of the freeway on these sections because of obstacles such as the median strip. The study also revealed that, after sixty minutes of driving, a driver's level of concentration begins to decrease. This suggests that taking a rest after sixty minutes of driving would be desirable and a safety campaign promoting this seems warranted.

The $\beta / \alpha$ measure seems to be more sensitive than the $P_{r e}$ measure in detecting differences in driving workload based on EEG signals. At least in the case of measuring 
driving workload on freeways, the EEG $\theta$ wave signal appears to be redundant, and this would suggest that the $\beta / \alpha$ ratio is the preferred measure.

This study has a limitation with respect to the gender and age distributions of test drivers. Some practical test conditions, such as attaching EEG gel pads on the hair, may have been considered unfavorable by the Korean women and resulted in a low number of female volunteers. Elderly drivers were purposely excluded from this particular study for safety concerns. For a follow-up study on elderly drivers, an indoor test with a driving simulator is considered to be the best option. A second limitation of this study is that it excluded the effects of lane changing behavior by asking drivers to only use the right-most travel lane in order to eliminate the effects of unknown external driving conditions. This restriction for reasons of scientific testing has brought about a certain degree of unrealistic driving.

Regardless of the above limitations, understanding the role of the visual and auditory effects on different types of road sections will provide opportunities to enhance freeway design (Orazio 2009; Heger 1998), to prepare safety campaigns, and eventually to reduce the potential causes of accidents. This study is limited to independent analyses on a driver's workload related to visual and auditory effects and elapsed driving time. For following studies, it is recommended that a combination study is conducted of visual and auditory effects on the elapsed driving time.

\section{Conflict of interest}

None of the authors have conflicting interests or financial disclosures.

\section{Acknowledgements}

This research was supported by the Ministry of Land, Infrastructure and Transport (MOLIT), Korea, under the Railway Technology Research Program (14RTRPB067916-02), supervised by the Korea Agency for Infrastructure Technology Advancement (KAIA). 


\section{References}

Bridger R. S., 1995. Introduction to Ergonomics. McGraw-Hill, New York.

Brookhuis, K.A., Waard, D., and Mulder, B. 1994. Measuring driving performance by car-following in traffic, Ergonomics, 37(3), 427-434.

Campbell, J.L. 2008. Human factors guidelines for road systems, NCHRP Report 600A.

Evans, L. 1991. Traffic safety and the driver. Van Nostrand Reinhold, New York.

Niedermeyer, E. and Silva, F. L. D. 2005. Electroencephalography, Basic principles clinical applications and related fields, fifth edition, Lippincott Williams \& Wilkins, Philadelphia.

Forbes, T.W. 1975. Human factors in highway traffic safety research. Wiley Interscience, New York.

Garber, N.J. and Hoel, L.A. 2009, Traffic \& Highway engineering, Fourth Edition, Cengage Learning.

Heger, R. 1998. Driving behavior and driver mental workload as criteria of highway geometric design quality, International symposium on highway geometric design practices, Transportation Research Circular, Issue Number E-C003, pp. 43:1-10.

Helander, M.G. 1975. Physiological reactions of drivers as indicators of road traffic demand. Transportation Research Record 530, pp. 1-17.

James, J.F. 2011. A Student's Guide to Fourier Transforms: With Applications in Physics and Engineering, Third Edition, Cambridge University Press, Cambridge.

Katsuki, H. 2003. Evaluation of mental load during driving by bio-signal analysis, IEIC Technical Report VOL 103, pp. 13-18.

Kim, J.Y. 1998. Evaluation of driver's psycho-physiological load at freeway merging area. Journal of Korean Society of Transportation 17 (2), pp. 69-79.

Korean National Police Agency, 2013. 2012 Road traffic accidents in Korea.

Lamm, R., Psarianos, B., and Mailaender, T. 1999. Highway design and traffic safety engineering handbook. McGraw-Hill, New York.

Orazio, P. 2009. An analysis of the effect of roadway design on driver's workload, the Baltic journal of road and bridge engineering, Volume 4(2) pp. 45-53.

Park, J.B., Chung, B.J., and Kim, J.Y. 1999. Development and installation of driver's behavior and response detecting system in vehicle. Korea Highway Cooperation. Highway Research Report 994(7-2), Seoul. 
Penfield, W. and Erickson, T.C. 1941. Epilepsy and cerebral localization, Oxford, England, Charles C. Thomas.

Scheuchenpflug, R. 2008. Psychological aspects of road construction: the self-explaining road, International Congress Transport Safety 2008.

Shin, Y.G., 1995. The effect to drivers workload between driving time and speed, Korea Road Traffic Authority.

Tijerina, L. 1996. Heavy vehicle driver workload assessment, Battelle Memorial Institute, National Highway Traffic Safety Administration.

Walter, W.G. 1953. The Living Brain. Norton, New York.

Wierwille, W.W., and Eggemeier, F.T. 1993. Recommendations for mental workload measurement in a test and evaluation environment. Human Factors 35-2, pp. 263-281. 
List of Figures

Fig. 1. EEG signals change in different relaxation/activation/drowsiness states

Fig. 2. DBDV and the data collection component system

Fig. 3. A participant driving the DBDV test unit

Fig. 4. EEG signal collection points (top view of a person's head)

Fig. 5. Visual effects analyzed with the selected measures

Fig. 6. Auditory effects analyzed with the selected measures

Fig. 7. Drivers' workload affected by elapsed travel times 


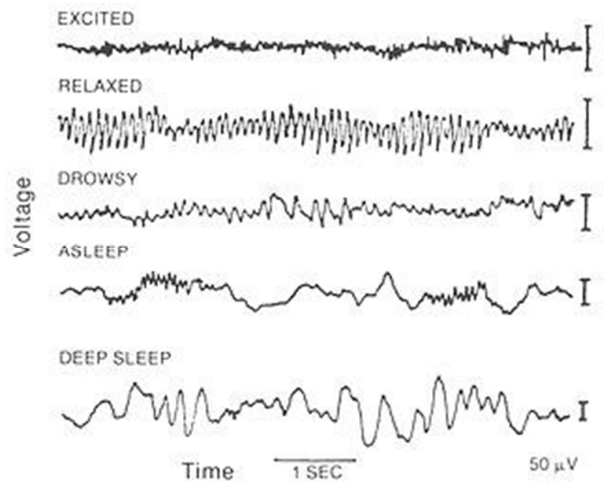

Fig. 1. EEG signals change in different relaxation/activation/drowsiness states

* Source: Penfield, W. and Erickson, T.C, Epilepsy and cerebral localization, Thomas, 1941 

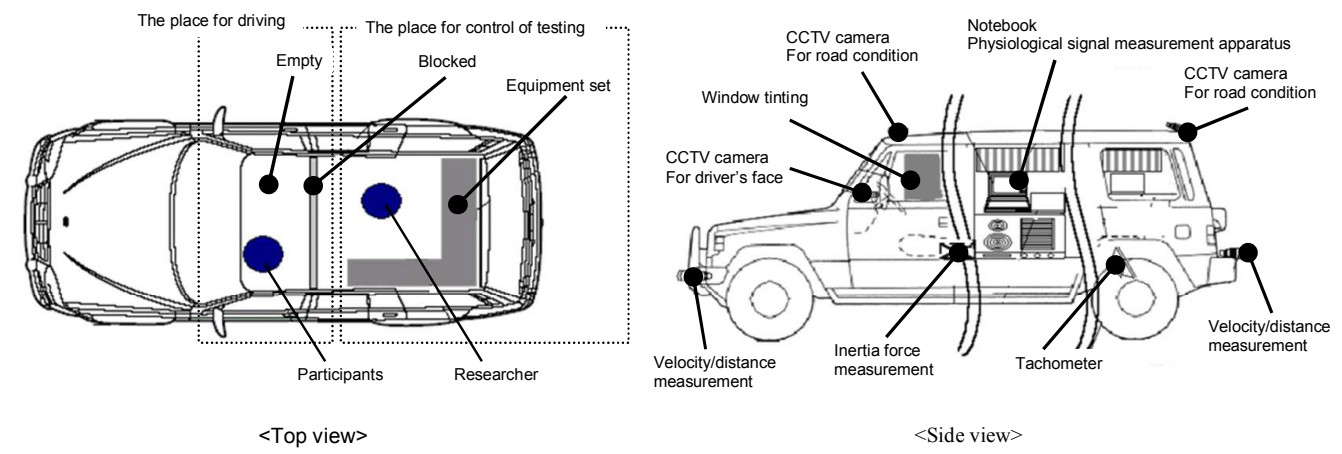

Fig. 2. DBDV and the data collection component system 


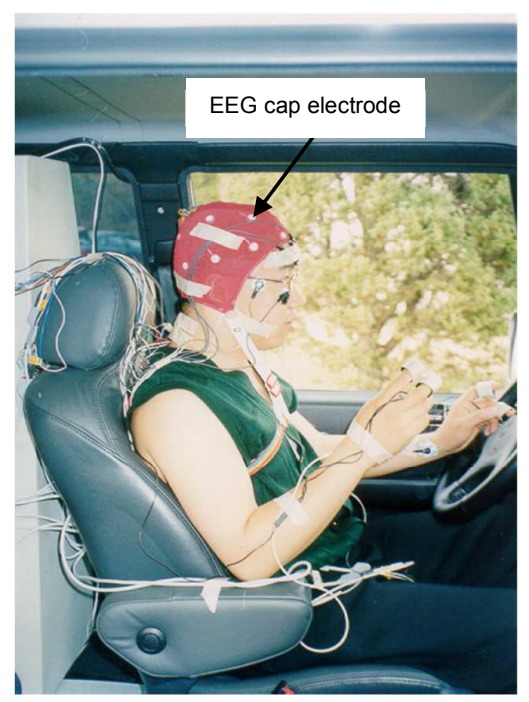

Fig. 3. A participant driving the DBDV test unit 


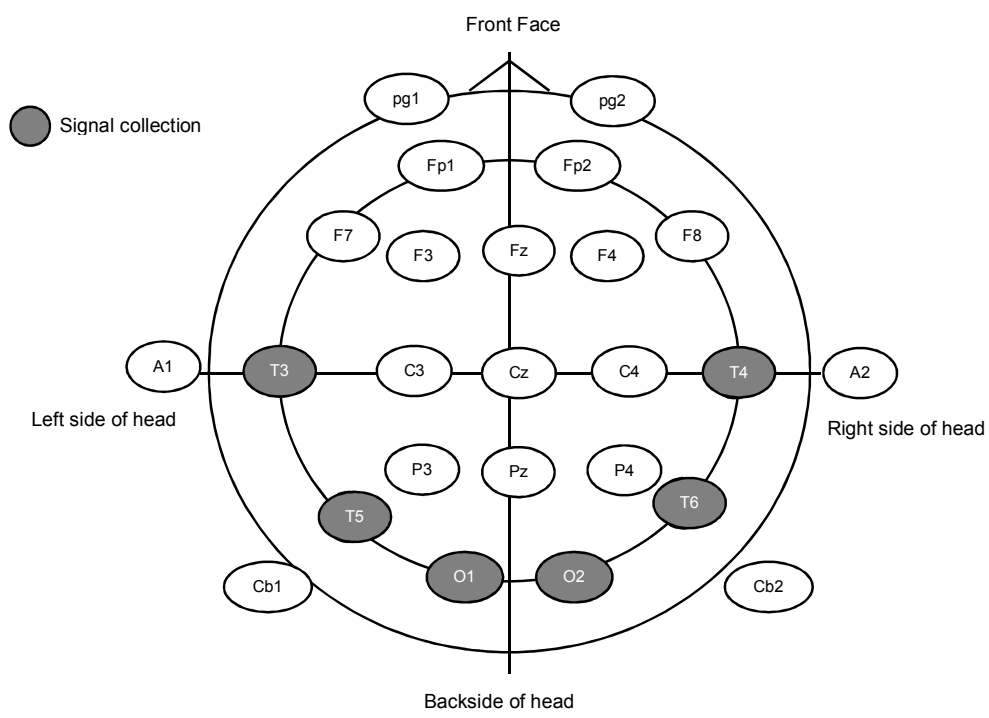

Fig. 4. EEG signal collection points (top view of a person's head) 


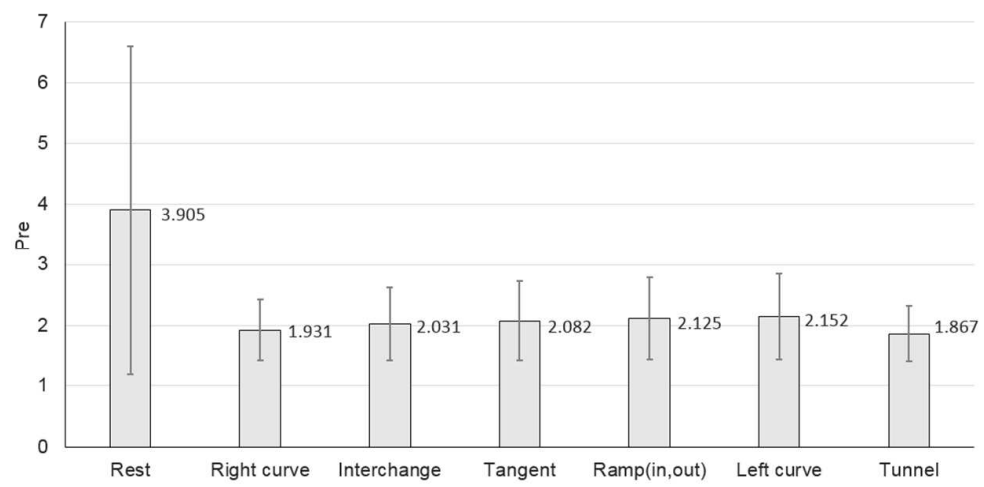

(a) The $P_{r e}$ measure

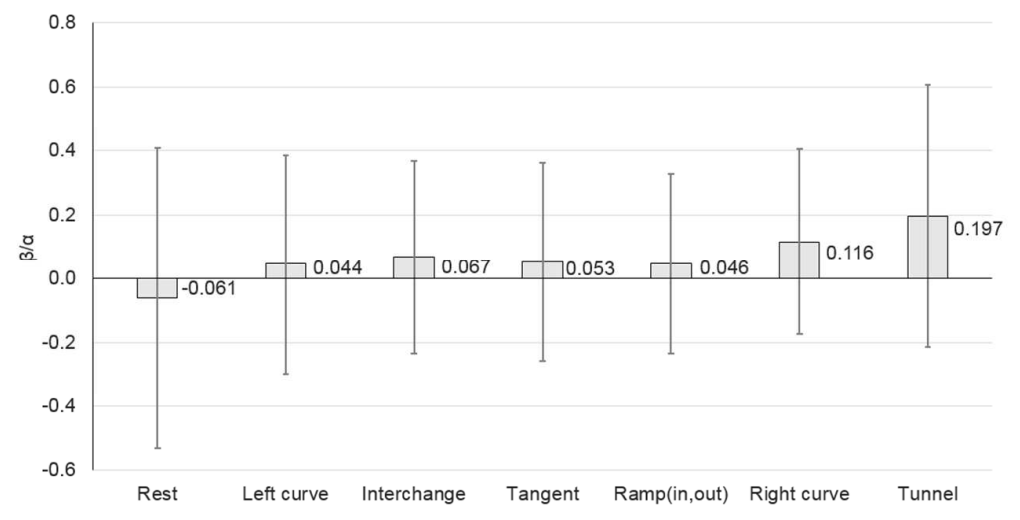

(b) The $\beta / \alpha$ measure

Fig. 5. Visual effects analyzed with the selected measures 


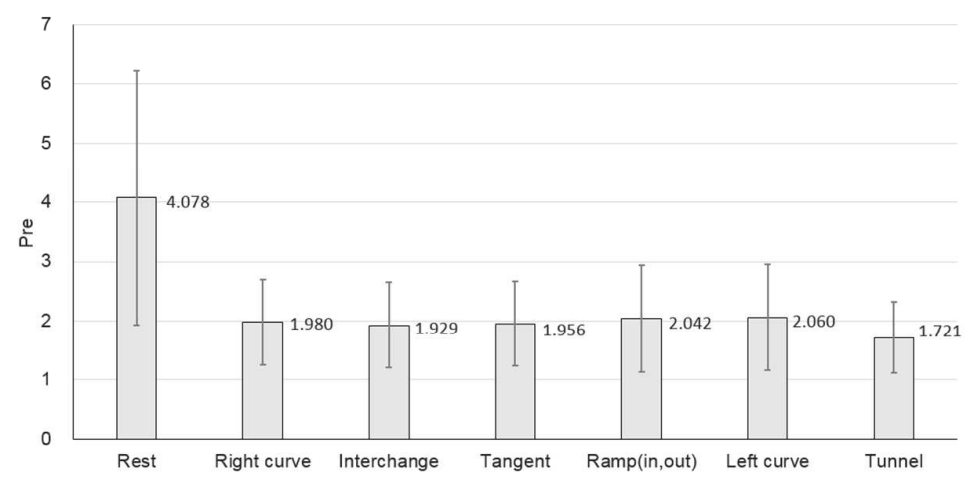

(a) The $P_{r e}$ measure

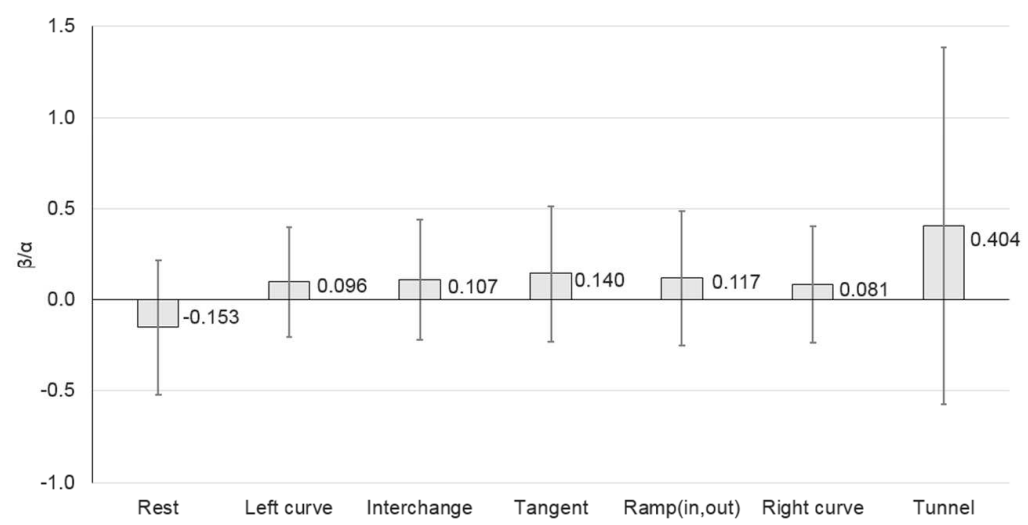

(b) The $\beta / \alpha$ measure

Fig. 6. Auditory effects analyzed with the selected measures 


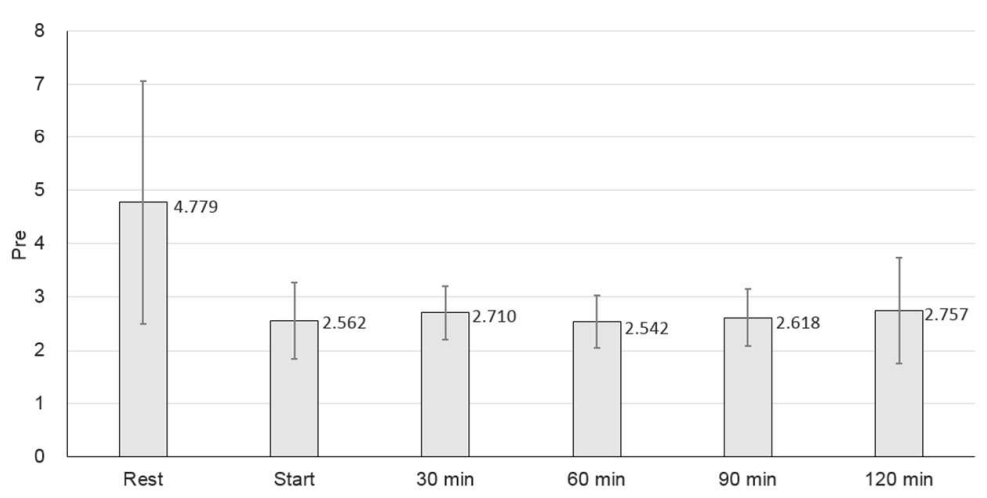

(a) The $P_{r e}$ measure

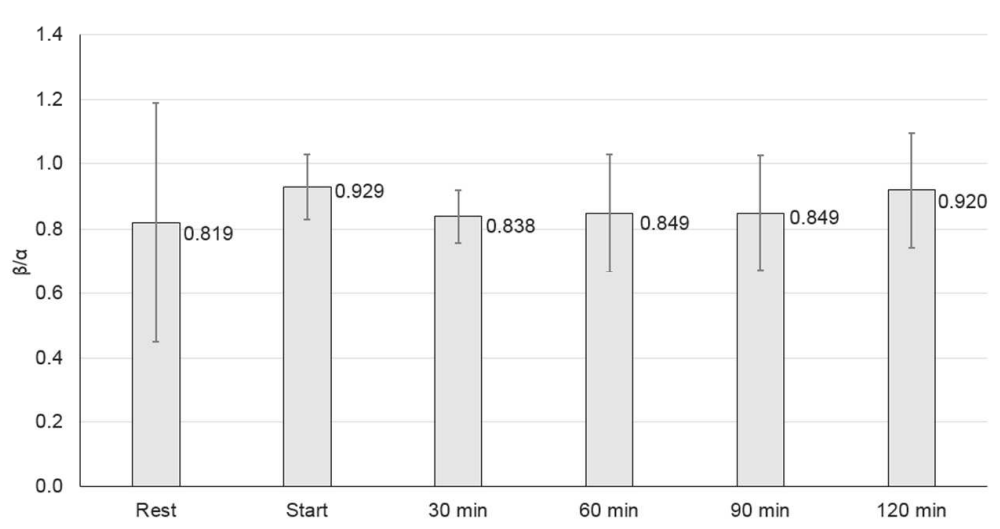

(b) The $\beta / \alpha$ measure

Fig. 7. Drivers' workload affected by elapsed travel times 
List of Tables

Table 1. Characteristics of the participants

Table 2. Freeway sections on which the driver's psychological responses were collected

Table 3. The average values of the EEG signals and the computed measures

Table 4. ANOVA results for the EEG signals from the occipital lobe (visual effects)

Table 5. Multiple ranges of the visual effects, grouped based on the measures

Table 6 ANOVA results for the EEG signals from the temporal lobe (auditory effects)

Table 7. Multiple ranges of the auditory effects, grouped based on the measures

Table 8. ANOVA results for the signals from the occipital lobe (effects of elapsed travel time)

Table 9. Multiple ranges of the elapsed travel times, grouped based on the $\beta / \alpha$ measure 
Table 1. Characteristics of the participants

\begin{tabular}{ccccccc}
\hline \multirow{2}{*}{ Age } & \multicolumn{2}{c}{ Number of participants } & & \multicolumn{4}{c}{ Average } \\
\cline { 2 - 3 } \cline { 5 - 6 } \cline { 5 - 6 } \cline { 5 - 6 } & Female & Male & & $\begin{array}{c}\text { Weight } \\
(\mathrm{Kg})\end{array}$ & $\begin{array}{c}\text { Height } \\
(\mathrm{Cm})\end{array}$ & $\begin{array}{c}\text { Driving Experience } \\
\text { (Year) }\end{array}$ \\
\hline $20 \sim 30$ & 5 & 26 & & 56.0 & 167.8 & 4.2 \\
$30 \sim 40$ & 3 & 10 & & 63.0 & 166.5 & 6.2 \\
40 over & 4 & 3 & & 62.8 & 165.5 & 6.9 \\
\hline
\end{tabular}


Table 2. Freeway sections on which the driver's psychological responses were collected

\begin{tabular}{cccc}
\hline $\begin{array}{c}\text { Freeway sections } \\
\text { for data collection }(\mathrm{km})\end{array}$ & $\begin{array}{c}\text { Buffer segments between } \\
\text { the subsections }(\mathrm{km})\end{array}$ & Facility types & \\
\hline 0.0 & N/A & - & - \\
$3.5 \sim 4.0$ & 3.5 & Tangent & 1 \\
$6.5 \sim 8.0$ & 2.5 & Tunnel & 1 \\
$13.0 \sim 13.5$ & 5.0 & Tangent & 2 \\
$17.0 \sim 17.5$ & 3.5 & Tunnel & 2 \\
$18.0 \sim 18.5$ & 0.5 & Ramp (in, out) & 1 \\
$23.5 \sim 24.5$ & 5.5 & Curve (right) & 1 \\
$30.5 \sim 32.5$ & 6.0 & Interchange & 1 \\
$37.5 \sim 38.5$ & 5.0 & Ramp (in, out) & 2 \\
$39.0 \sim 39.5$ & 0.5 & Tangent & 3 \\
$43.0 \sim 43.5$ & 3.5 & Curve (right) & 2 \\
$45.0 \sim 47.5$ & 1.5 & Curve (left) & 1 \\
$60.5 \sim 61.0$ & 13.0 & Tunnel & 3 \\
$62.0 \sim 63.5$ & 1.0 & Curve (left) & 2 \\
$72.0 \sim 73.0$ & 8.5 & Ramp (in, out) & 3 \\
$83.0 \sim 84.5$ & 10.0 & Curve (left) & 3 \\
$87.0 \sim 88.0$ & 2.5 & Curve (right) & 3 \\
88.0 & N/A & Turning point & - \\
\hline
\end{tabular}


Table 3. The average values of the EEG signals and the computed measures

\begin{tabular}{|c|c|c|c|c|c|c|c|c|}
\hline \multicolumn{2}{|c|}{ Section } & $\begin{array}{c}\text { Rest } \\
\text { (no driv.) }\end{array}$ & Tangent & $\begin{array}{l}\text { Curve } \\
\text { (left) }\end{array}$ & $\begin{array}{l}\text { Curve } \\
\text { (right) }\end{array}$ & $\begin{array}{l}\text { Ramp } \\
\text { (in, out) }\end{array}$ & Interchange & Tunnel \\
\hline \multirow{5}{*}{$\begin{array}{c}\text { Occipital } \\
\text { lobe } \\
\text { (visual } \\
\text { effects) }\end{array}$} & $\alpha_{\text {spectrum }}$ & 0.105 & 0.122 & 0.121 & 0.124 & 0.119 & 0.132 & 0.121 \\
\hline & $\beta_{\text {spectrum }}$ & 0.094 & 0.127 & 0.127 & 0.137 & 0.126 & 0.141 & 0.141 \\
\hline & $\theta_{\text {spectrum }}$ & 0.262 & 0.143 & 0.153 & 0.141 & 0.150 & 0.155 & 0.142 \\
\hline & $P_{r e}$ & 3.905 & 2.082 & 2.152 & 1.932 & 2.125 & 2.031 & 1.867 \\
\hline & $\beta / \alpha$ & -0.060 & 0.053 & 0.044 & 0.116 & 0.046 & 0.067 & 0.197 \\
\hline \multirow{5}{*}{$\begin{array}{c}\text { Temporal } \\
\text { lobe } \\
\text { (auditory } \\
\text { effects) }\end{array}$} & $\alpha_{\text {spectrum }}$ & 0.119 & 0.129 & 0.125 & 0.124 & 0.127 & 0.135 & 0.121 \\
\hline & $\beta_{\text {spectrum }}$ & 0.098 & 0.146 & 0.134 & 0.137 & 0.144 & 0.152 & 0.148 \\
\hline & $\theta_{\text {spectrum }}$ & 0.279 & 0.157 & 0.151 & 0.146 & 0.167 & 0.159 & 0.133 \\
\hline & $P_{r e}$ & 4.077 & 1.956 & 2.060 & 1.980 & 2.042 & 1.929 & 1.721 \\
\hline & $\beta / \alpha$ & -0.152 & 0.140 & 0.081 & 0.096 & 0.117 & 0.107 & 0.104 \\
\hline
\end{tabular}


Table 4. ANOVA results for the EEG signals from the occipital lobe (visual effects)

\begin{tabular}{ccccccc}
\hline Measurement & $d f$ & SS & MS & F value $(\alpha=0.05)$ & $P_{r}>\mathrm{F}$ & $H_{0}$ \\
\hline$P_{r e}$ & 6 & 61.1754 & 10.1959 & 6.24 & 0.0001 & Reject \\
$\beta / \alpha$ & 6 & 0.9663 & 0.1611 & 2.52 & 0.0294 & Reject \\
\hline
\end{tabular}


Table 5. Multiple ranges of the visual effects, grouped based on the measures

\begin{tabular}{lccccc}
\hline Facility & \multicolumn{2}{c}{$\begin{array}{c}\text { Duncan's groups } \\
\text { based on the } \boldsymbol{P}_{r e}\end{array}$} & & \multicolumn{2}{c}{$\begin{array}{c}\text { Duncan's groups } \\
\text { based on the } \boldsymbol{\beta} / \boldsymbol{\alpha} \text { measure }\end{array}$} \\
\cline { 3 - 3 } \cline { 5 - 6 } Rest (not driving) & $\mathrm{A}$ & - & $\mathrm{A}$ & - \\
Right curve & - & $\mathrm{B}$ & $\mathrm{B}$ & $\mathrm{A}$ & $\mathrm{B}$ \\
Interchange & - & $\mathrm{B}$ & & $\mathrm{A}$ & $\mathrm{B}$ \\
Tangent & - & $\mathrm{B}$ & $\mathrm{A}$ & $\mathrm{B}$ \\
Ramp (in, out) & - & $\mathrm{B}$ & $\mathrm{A}$ & $\mathrm{B}$ \\
Left curve & - & $\mathrm{B}$ & & - & $\mathrm{B}$ \\
Tunnel & - & - & - & $\mathrm{B}$ \\
\hline
\end{tabular}


Table 6. ANOVA results for the EEG signals from the temporal lobe (auditory effects)

\begin{tabular}{ccccccc}
\hline Measurement & $d f$ & SS & MS & F value $(\alpha=0.05)$ & $P_{r}>\mathrm{F}$ & $H_{0}$ \\
\hline$P_{r e}$ & 6 & 58.2569 & 9.7095 & 13.53 & 0.0001 & Reject \\
$\beta / \alpha$ & 6 & 4.0636 & 0.6773 & 4.04 & 0.0001 & Reject \\
\hline
\end{tabular}


Table 7. Multiple ranges of the auditory effects, grouped based on the measures

\begin{tabular}{|c|c|c|c|c|c|}
\hline Facility & \multicolumn{2}{|c|}{ Duncan’s group } & \multicolumn{3}{|c|}{$\begin{array}{c}\text { Duncan's groups } \\
\text { based on the } \boldsymbol{\beta} / \boldsymbol{\alpha} \text { measure }\end{array}$} \\
\hline Rest (not driving) & $\mathrm{A}$ & - & $\mathrm{A}$ & - & - \\
\hline Right curve & - & $\mathrm{B}$ & - & $\mathrm{B}$ & - \\
\hline Interchange & - & $\mathrm{B}$ & - & $\mathrm{B}$ & - \\
\hline Tangent & - & $\mathrm{B}$ & - & B & - \\
\hline Ramp (in, out) & - & $\mathrm{B}$ & - & B & - \\
\hline Left curve & - & $\mathrm{B}$ & - & B & - \\
\hline Tunnel & - & $\mathrm{B}$ & - & - & $\mathrm{C}$ \\
\hline
\end{tabular}


Table 8. ANOVA results for the signals from the occipital lobe (effects of elapsed travel time)

\begin{tabular}{ccccccc}
\hline Measurement & $d f$ & SS & MS & F value $(\alpha=0.05)$ & $P_{r}>\mathrm{F}$ & $H_{0}$ \\
\hline$P_{r e}$ & 5 & 13.7502 & 2.7500 & 1.72 & 0.1680 & Not reject \\
$\beta / \alpha$ & 5 & 0.6327 & 0.1265 & 2.90 & 0.0347 & Reject \\
\hline
\end{tabular}


Table 9. Multiple ranges of the elapsed travel times, grouped based on the $\beta / \alpha$ measure

\begin{tabular}{lccc}
\hline Elapsed travel time & & Duncan's group \\
\cline { 1 - 1 } \cline { 3 - 4 } Rest (not driving) & & $\mathrm{A}$ & - \\
Start driving & - & $\mathrm{B}$ \\
$0 \sim 30$ minutes & $\mathrm{A}$ & $\mathrm{B}$ \\
$30 \sim 60$ minutes & $\mathrm{A}$ & $\mathrm{B}$ \\
$60 \sim 90$ minutes & - & $\mathrm{B}$ \\
$90 \sim 120$ minutes & - & $\mathrm{B}$ \\
\hline
\end{tabular}

